\title{
Optomechanical Cooling of a Macroscopic Oscillator by Homodyne Feedback
}

\author{
Stefano Mancini, David Vitali and Paolo Tombesi \\ Dipartimento di Matematica e Fisica, Università di Camerino, I-62032 Italy \\ and Istituto Nazionale di Fisica della Materia, Italy
}

(Received: February 15, 2018)

\begin{abstract}
We propose a simple optomechanical model in which a mechanical oscillator quadrature could be "cooled" well below its equilibrium temperature by applying a suitable feedback to drive the orthogonal quadrature by means of the homodyne current of the radiation field used to probe its position.
\end{abstract}

PACS numbers(s): 03.65.Bz, 42.50.Dv, 42.50.Vk

The problem of considering a macroscopic oscillator in terms of Quantum Mechanics is usually avoided because one can obtain the right results without using any quantum mechanical hypothesys. When, however, one whishes to use it as a device to detect extremely small displacements due to very weak forces, as in the gravitational wave detectors, one has to be careful in considering it as a mere macroscopic object. Should one consider a macroscopic oscillator as a quantum oscillator, once all other possible noise sources were eliminated by using filters, screens, insulators etc., the ultimate criterion one has to satisfy is the one associated with the thermal noise [1.2]. For the harmonic oscillator it means $k_{B} T<\hbar \omega_{m} / 2$, where $k_{B}$ is the Boltzmann constant, $\omega_{m}$ the mechanical angular frequency and $T$ the temperature of the environment in which the oscillator lives. This prohibitive limit, for macroscopic massive oscillators, is however only valid when the measurement time $\tau$ is of the order of the mechanical relaxation time $\tau_{m}$. The actual limit can be expressed as $2 k_{B} T \tau / Q_{m}<\hbar$ [1]. In this case it is possible to consider a macroscopic mechanical oscillator as a quantum oscillator even at liquid $\mathrm{He}$ temperature [3], but very high mechanical $Q_{m}=\omega_{m} \tau_{m}$ factors and also short observation times should be considered. To have better results and, for instance, to detect millisecond duration bursts of gravitational waves from supernovae, one should measure out of resonance, as in VIRGO or LIGO proposals [4], or at lower temperatures, as in massive bar detector schemes [5]. The thermal fluctuations are, however, the fundamental limitations and, in order to reduce their effects, one usually should lower the environment's temperature.

In this letter we present an alternative way of cooling the oscillator's observed quadrature which could be experimentally accessible.

We consider an empty Fabry-Perot cavity with one fixed mirror with transmittivity $T_{r}$ and one perfectly reflecting end mirror. The completely reflecting mirror can move, undergoing harmonic oscillations damped by the coupling to a thermal bath in equilibrium at temperature $T$. The cavity resonances are calculated in the absence of the impinging field, hence, if $L$ is the equilibrium cavity length, the resonant frequency of the cavity will be 
$\nu_{c}=\omega_{c} / 2 \pi=n c / 2 L$, where $n$ is an arbitrary integer number and $c$ the speed of light. Furthermore, we assume that at the frequency of the impinging field $\nu_{0}$, the fixed mirror does not introduce any excess noise beyond the input field noise. We also assume that retardation effects, due to the oscillating mirror in the intracavity field, are negligible. We shall use a field intensity such that the correction to the radiation pressure force, due to the Doppler frequency shift of the photons [6] on the moving mirror, is completely negligible. This means considering the damping coefficient of the oscillating mirror to be only due to the coupling with the thermal bath. Thus, we can write the Hamiltonian as

$$
H=\hbar \omega_{c}\left(B^{\dagger} B+\frac{1}{2}\right)+\frac{\hat{p}^{2}}{2 m}+\frac{1}{2} m \omega_{m}^{2} \hat{x}^{2}+H_{i n t},
$$

where $B$ and $B^{\dagger}$ are the boson operators of the resonant cavity mode; $\hat{p}$ and $\hat{x}$ are the momentum and the displacement operators, respectively, from the equilibrium position of the oscillating mirror with mass $m$ and oscillation frequency $\nu_{m}=\omega_{m} / 2 \pi$. The mechanical angular frequency $\omega_{m}$ will be many orders of magnitude smaller than $\omega_{c}$ to ensure that the number of photons generated by the Casimir effect [7] is completely negligible; we actually are in the so called adiabatic approximation, i.e., the cavity round trip time of the photon is much shorter than the mirror's period of oscillation. $H_{\text {int }}$ accounts for the interaction between the cavity mode and the oscillating mirror 8. Since we have assumed no retardation effects, $H_{\text {int }}$ simply represents the effect of the radiation pressure force which causes the instantaneous displacement $\hat{x}$ of the mirror [9, 10], and can be written as

$$
H_{i n t}=-\hbar \frac{\omega_{c}}{L} B^{\dagger} B \hat{x}=-\hbar G B^{\dagger} B\left(A+A^{\dagger}\right),
$$

where we have introduced the dimensionless ladder operators $\left(A\right.$ and $\left.A^{\dagger}\right)$ for the oscillating mirror, and the coupling constant becomes $G=\sqrt{\hbar \omega_{c}^{2} / 2 m \omega_{m} L^{2}}$. The intracavity radiation field mode $B$ is damped through the output fixed mirror at a rate $\gamma_{b}=c T_{r} / 2 L$, while $\gamma_{m}$ is the mechanical damping rate $\left(\gamma_{m}<<\gamma_{b}\right)$.

The above interaction (2) gives rise to non linear stochastic equations whose linearization around the steady state is equivalent, in a frame rotating at the impinging frequency $\nu_{0}$, to replace Eq. (1) with [9]

$$
H^{\prime}=\hbar \Delta b^{\dagger} b+\hbar \omega_{m} a^{\dagger} a+H_{i n t}^{\prime},
$$

where now all the operators represent small fluctuations around steady state values, i.e. $B=\beta_{s}+b$ and $A=\langle A\rangle_{s}+a$. These are determined by $x_{s}=$ $\sqrt{\hbar / 2 m \omega_{m}}\left(\langle A\rangle_{s}+\langle A\rangle_{s}^{*}\right)=\hbar \omega_{c}\left|\beta_{s}\right|^{2} / m \omega_{m}^{2} L ; \beta_{s}=$ $\langle B\rangle_{s}=\sqrt{\gamma_{b}} \beta_{i n} /\left(\frac{\gamma_{b}}{2}-i \Delta\right)$, with $\beta_{i n}$ the classical field characterizing the input laser power $P_{\text {in }}=\hbar \omega_{0}\left|\beta_{i n}\right|^{2}$. The parameter $\Delta$ represents the radiation phase shift due to the detuning and to the stationary displacement of the mirror, $\Delta=\omega_{c}-\omega_{0}-\omega_{c} x_{s} / L$; further we have 


$$
H_{i n t}^{\prime}=\hbar \chi X Y_{\varphi}
$$

where $X=\left(a+a^{\dagger}\right) / 2$ is the mirror position quadrature, $Y_{\varphi}=\left(b e^{i \varphi}+b^{\dagger} e^{-i \varphi}\right) / 2$ is the radiation quadrature with $\varphi=\arg \left(\beta_{s}\right)$, and $\chi=-4 G\left|\beta_{s}\right|$. Eqs. (3) and (4) represent the starting point for further analysis of our system.

The mirror displacement induces a phase shift on the radiation field, hence the latter can be used as a "meter" to measure the mirror position quadrature $X$. For simplicity, in Eq. (3) we assume we can set $\Delta=0$ by just varying the cavity detuning. This setting allows us to write the evolution equation for the whole density operator $D$ as

$$
\dot{D}=\mathcal{L} D-\frac{i}{\hbar}\left[H_{\text {int }}^{\prime}, D\right]+\frac{\gamma_{b}}{2}\left(2 b D b^{\dagger}-b^{\dagger} b D-D b^{\dagger} b\right)
$$

where $\mathcal{L}$ describes the damped dynamics of the mechanical mode $a$ which is considered in thermal equilibrium at temperature $T$. We also assume that the number of thermal photons is negligibly small at optical frequency. Following the treatment of Refs. [11,12], we assume that the radiation mode $b$ is heavily damped, so that due to the large value of $\gamma_{b}>>|\chi|$, the $b$ mode always will be near to its vacuum state (we are considering fluctuations around the steady state $\beta_{s}$ ). This allows us to adiabatically eliminate the $b$ mode and to perform a perturbative calculation in the small parameter $\chi / \gamma_{b}$ [13].

The measurement of the quadrature $X$ is obtained by performing a homodyne measurement [14] of a generic quadrature of the "meter" mode $b$, i.e. $Y_{-\delta}=$ $\left(b e^{-i \delta}+b^{\dagger} e^{i \delta}\right) / 2$ with $\delta$ a phase connected to the local oscillator [14]. Due to the interaction (4) between the two modes, one gets information on $X$ by directly observing the mode $b$. The continuous monitoring of the $b$ mode via homodyne detection modifies the time evolution of the whole system.

We are now able to apply a phase-dependent feedback loop to control the dynamics of the mechanical mode of interest $a$. Other feedback schemes could be devised [15], but only the phase-dependent feedback produces the desired effect. In order to be easily followed, we use the continous feedback theory recently proposed by Wiseman and Milburn [16], who well explained the implications and limitations of this feedback. One has to take part of the stochastic output homodyne photocurrent [17] obtained from the continuous monitoring of the meter mode $b$, and feed it back to the mirror dynamics (for example as a driving term) in order to modify the evolution of the mode $a$.

In the limiting case of a feedback delay time much shorter than the characteristic time of the mechanical mode, it is possible to obtain a Markovian equation for the reduced density matrix $\rho=\operatorname{Tr}_{b} D$ in the presence of feedback [16. Thus, following Ref. [16], we get 
$\dot{\rho}=\mathcal{L} \rho-\frac{\Gamma}{2}[X,[X, \rho]]+\mathcal{K}\left(i e^{i \phi} \rho X-i e^{-i \phi} X \rho\right)+\frac{\mathcal{K}^{2}}{2 \eta \Gamma} \rho$

where $\Gamma=\chi^{2} / \gamma_{b}$ (we have defined $\phi=\delta+\varphi$, which is the only relevant phase of the $b$ mode influencing the dynamics of the mechanical mode $a$ ), $\mathcal{K}$ is a Liouville superoperator describing the way in which the feedback signal acts on the system of interest and $\eta$ represents the photodetector efficiency. This master equation is the starting point of our discussion. The second term of the right hand side of Eq. (6) is the usual double-commutator term associated to the measurement of $X$, it results from the elimination of the radiation variables; the third term is the feedback term itself and the fourth term is a diffusion-like term, which is an unavoidable consequence of the noise introduced by the feedback itself.

The mirror is considered to be in a thermal bath characterized by a damping constant $\gamma_{m}$, so that we have

$$
\begin{aligned}
\mathcal{L} \rho & =-i \omega_{m}\left[a^{\dagger} a, \rho\right]+\frac{\gamma_{m}}{4}\left[a+a^{\dagger},\left[a^{\dagger}-a, \rho\right]_{+}\right] \\
& -\frac{\gamma_{m}}{2} \frac{k_{B} T}{\hbar \omega_{m}}\left[a+a^{\dagger},\left[a+a^{\dagger}, \rho\right]\right]
\end{aligned}
$$

where $[,]_{+}$means the anticommutator and the limit $k_{B} T>>\hbar \omega_{m}$ is taken into account [18], and due to the frequency we are considering, is surely valid at room temperature down to millikelvins at least. Moreover, since the Liouville superoperator $\mathcal{K}$ can only be of Hamiltonian form [16], we choose it as $\mathcal{K} \rho=g\left[a-a^{\dagger}, \rho\right] / 2$ [11,12, which means feeding back the measured homodyne photocurrent to the mechanical oscillator with a driving term in the Hamiltonian involving the mechanical quadrature orthogonal to the measured one; $g$ is the feedback gain related to the practical way of realizing the loop. One could have chosen to feed the system with a generic phase-dependent quadrature, due to the homodyne current, however, it will turn out that the above choice gives the best and simplest result. Since the measured quadrature of the mirror is its position the feedback will act as a drive for the momentum. Using the above expressions in Eq. (6) and rearranging the terms in an appropriate way, we finally get the following master equation:

$$
\begin{aligned}
\dot{\rho} & =\frac{\gamma}{2}(N+1)\left(2 a \rho a^{\dagger}-a^{\dagger} a \rho-\rho a^{\dagger} a\right) \\
& +\frac{\gamma}{2} N\left(2 a^{\dagger} \rho a-a a^{\dagger} \rho-\rho a a^{\dagger}\right) \\
& -\frac{\gamma}{2} M\left(2 a^{\dagger} \rho a^{\dagger}-a^{\dagger 2} \rho-\rho a^{\dagger 2}\right) \\
& -\frac{\gamma}{2} M^{*}\left(2 a \rho a-a^{2} \rho-\rho a^{2}\right)-i \omega_{m}\left[a^{\dagger} a, \rho\right] \\
& -\left(\frac{g}{4} \sin \phi+\frac{\gamma_{m}}{4}\right)\left(\left[a^{2}, \rho\right]-\left[a^{\dagger 2}, \rho\right]\right),
\end{aligned}
$$

where $\gamma=\gamma_{m}-g \sin \phi$, and 


$$
\begin{aligned}
N & =\frac{1}{\gamma}\left[\gamma_{m}\left(\frac{k_{B} T}{\hbar \omega_{m}}-\frac{1}{2}\right)+\frac{\Gamma}{4}+\frac{g^{2}}{4 \eta \Gamma}+\frac{g}{2} \sin \phi\right] \\
M & =-\frac{1}{\gamma}\left[\gamma_{m} \frac{k_{B} T}{\hbar \omega_{m}}+\frac{\Gamma}{4}-\frac{g^{2}}{4 \eta \Gamma}-i \frac{g}{2} \cos \phi\right] .
\end{aligned}
$$

This Eq. (8) is very instructive because it clearly shows the effects of the feedback loop on the mirror mode a. The proposed feedback mechanism, indeed, not only introduces a driving term to the mirror's momentum quadrature, it also simulates the presence of a bath with nonstandard fluctuations, characterized by an effective damping constant $\gamma$ and by the coefficients $M$ and $N$, which are given in terms of the feedback parameters [12]. For the positivity of the density matrix the external parameters should be chosen such that $|M|^{2}<N(N+1)$. This can be checked with a unitary transformation giving Eq. (8) in a manifest Lindblad form [19] for the above inequality. An interesting aspect of the effective bath described by the first four terms in the right hand side of (8) is that it is characterized by phase-sensitive fluctuations, depending upon the experimentally adjustable phase $\phi$.

Because of its linearity, the solution of Eq. (8) can be easily obtained, as shown in Refs. [11], by using the normally ordered characteristic function [20] and assuming the mirror initially in a thermal state at temperature $T$, i.e. $\rho(0)=\left(1-e^{-\hbar \omega_{m} / k_{B} T}\right) \sum_{n}|n\rangle\langle n| e^{-n \hbar \omega_{m} / k_{B} T}$, where $|n\rangle$ is the number state of the mode $a$.

The stationary state is reached only if the parameters $g, \phi, \omega_{m}$ and $\gamma_{m}$ satisfy the stability conditions $\gamma_{m}-$ $g \sin \phi>0$ and $\omega_{m}^{2}-\gamma_{m} g \sin \phi>0$. For simplicity we choose $\phi=-\pi / 2$ from now on since this choice turns out to be best. Under the stability conditions and in the long time limit $(t \rightarrow \infty)$ the variance of the position quadrature operator $X=\left(a+a^{\dagger}\right) / 2$ for the mirror is

$$
\begin{aligned}
& \left\langle X^{2}\right\rangle=\frac{g^{2}}{8 \eta \Gamma} \frac{\gamma_{m}^{2}+\omega_{m}^{2}+\gamma_{m} g}{\left(\gamma_{m}+g\right)\left(\omega_{m}^{2}+\gamma_{m} g\right)} \\
& +\left(\frac{k_{B} T}{2 \hbar \omega_{m}}+\frac{\Gamma}{8 \gamma_{m}}\right) \frac{\gamma_{m} \omega_{m}^{2}}{\left(\gamma_{m}+g\right)\left(\omega_{m}^{2}+\gamma_{m} g\right)},
\end{aligned}
$$

while for the orthogonal quadrature $P=\left(a-a^{\dagger}\right) /(2 i)$, i.e. the mirror's momentum, we get

$$
\begin{aligned}
& \left\langle P^{2}\right\rangle=\frac{g^{2}}{8 \eta \Gamma} \frac{\omega_{m}^{2}}{\left(\gamma_{m}+g\right)\left(\omega_{m}^{2}+\gamma_{m} g\right)} \\
& +\left(\frac{k_{B} T}{2 \hbar \omega_{m}}+\frac{\Gamma}{8 \gamma_{m}}\right) \gamma_{m} \frac{g^{2}+\omega_{m}^{2}+\gamma_{m} g}{\left(\gamma_{m}+g\right)\left(\omega_{m}^{2}+\gamma_{m} g\right)} .
\end{aligned}
$$

In the case of no coupling with the cavity mode, the above variances for the macroscopic oscillator only consist in the thermal noise as one should expect. Whenever an indirect detection of the mirror position is made the backaction noise is added. The latter, however, is usually negligibly small compared with the previous one. Instead, by using the feedback with sufficient high gain 
$\left(g>>\omega_{m} Q_{m}\right)$, we can set $T_{\text {eff }} \approx T \omega_{m}^{2} / g^{2}$ as an effective temperature, and the mirror's position quadrature variance becomes

$$
\left\langle X^{2}\right\rangle \approx \frac{k_{B} T_{e f f}}{2 \hbar \omega_{m}}+\frac{\Gamma \omega_{m}^{2}}{8 \gamma_{m} g^{2}}+\frac{g}{8 \eta \Gamma} .
$$

Although the feedback introduces excess noise, it also gives a scale factor for the thermal noise term by means of $T_{\text {eff }}$, so that with an appropriate choice of the parameters, the latter can be strongly reduced.

It is also to remark that the proposed phase-dependent feedback does not produce a proper squeezing; moreover, it can extract the thermal noise from the system, because the variance reduction occurs, for not extremely high values of $g$, in both quadratures, as can be evicted from Eqs. $(9$, 10). Hence, it acts as a refrigerator.

To better show the potentiality of this feedback mechanism let us consider the spectrum of the position quadrature. To this end the Fourier transforms of the stochastic equations connected with the master equation (8) are easily written down 21

$$
\begin{aligned}
i \omega \tilde{X}(\omega) & =\omega_{m} \tilde{P}(\omega)-g \tilde{X}(\omega)-\sqrt{\gamma} \tilde{X}_{i n}(\omega) \\
i \omega \tilde{P}(\omega) & =-\omega_{m} \tilde{X}(\omega)-\gamma_{m} \tilde{P}(\omega)-\sqrt{\gamma} \tilde{P}_{i n}(\omega)
\end{aligned}
$$

and the input noise operators have the following correlations

$$
\begin{aligned}
\left\langle\tilde{X}_{i n}(\omega) \tilde{X}_{i n}\left(-\omega^{\prime}\right)\right\rangle & =\frac{1}{4}(2 N+1+2 \operatorname{Re}\{M\}) \delta\left(\omega-\omega^{\prime}\right) \\
\left\langle\tilde{P}_{i n}(\omega) \tilde{P}_{i n}\left(-\omega^{\prime}\right)\right\rangle & =\frac{1}{4}(2 N+1-2 \operatorname{Re}\{M\}) \delta\left(\omega-\omega^{\prime}\right) \\
\left\langle\tilde{X}_{i n}(\omega) \tilde{P}_{i n}\left(-\omega^{\prime}\right)\right\rangle & =\frac{1}{4}(i+2 \operatorname{Im}\{M\}) \delta\left(\omega-\omega^{\prime}\right) .
\end{aligned}
$$

Defining $S_{g}(\omega)=\langle\tilde{X}(\omega) \tilde{X}(-\omega)\rangle_{s}$, we get from Eqs. (12)

$$
\begin{aligned}
S_{g}(\omega) & =\frac{\gamma}{4} \frac{1}{|\Xi(\omega)|^{2}}\left[\left(\gamma_{m}^{2}+\omega^{2}+\omega_{m}^{2}\right)(2 N+1)\right. \\
& \left.+\left(\gamma_{m}^{2}+\omega^{2}-\omega_{m}^{2}\right) 2 \operatorname{Re}\{M\}\right],
\end{aligned}
$$

where the subscript $s$ indicates the symmetrized correlation and $\Xi(\omega)=\left[(i \omega+g)\left(i \omega+\gamma_{m}\right)+\omega_{m}^{2}\right]$.

As a practical example we take the physical parameters of the model presented in Ref. [10]. Taking their values for granted, i.e. $m=10 \mathrm{Kg}, \nu_{m}=10 \mathrm{~Hz}, \gamma_{m}=1 \mathrm{~s}^{-1}$, $L=4 \mathrm{~m}, \nu_{0}=5.82 \times 10^{14} \mathrm{~Hz}, T_{r}=0.02, P_{\text {in }}=10 \mathrm{~W}$, we get $\Gamma \approx 200 \mathrm{~s}^{-1}$, then $\chi \approx 10^{4} \mathrm{~s}^{-1}$. This choice satisfies the relation $\gamma_{b}>>\chi$ and all others inequalities, and we further take $\eta \approx 1$ and $T=300 \mathrm{~K}$.

Then, in Fig. 1 we show the (scaled) spectrum of Eq. (14) for various values of $g$. The curve for $g=0$ practically coincides with the spectrum of the mirror not coupled to the cavity mode because of the smallness of the 
backaction noise. It results evident that for high values of the feedback gain the spectrum is practically vanishing while the peak at the mechanical resonance frequency gradually disappears and one peak at zero frequency appears, with very small amplitude. With the proposed feedback, a transition from a dissipative to a diffusive behaviour of the oscillator is obtained by just varying the feedback gain. These results, although in a different context, are similar, but not equivalent, to those obtained in Ref. [15], where the feedback was used for the regulation of a microcantilever response and a direct photodetection was used, instead of our phase-dependent scheme. Furthermore, the temperature of the bath was assumed negligibly small.

Summarizing, we have proposed a feedback scheme based on an indirect measurement to reduce position quadrature uncertainty of a macroscopic oscillator. The described mechanism can be very useful in reducing the effect of thermal noise in quadratures of macroscopic mirrors, as those devoted to the gravitational wave detectors, even at room temperatures. The feedback loop may consist in a transducer [2] which transforms the random optical signal in a stochastic electric signal which in turn acts as a mechanical driving on the mirror's momentum. This could be readly realized, but it is not the only way, by using the feedback current to vary the potential of a capacitor formed by the oscillating mirror and a fixed plate. On the other hand, depending on the specific experimental realization of the feedback loop there could be some limitations on the values of $g$.

We think that the practical implementation of the discussed model, even though in a situation far from the oversimplified theoretical one, should be an interesting challenge for an experimentalist, and it will turn out extremely useful in reducing the thermal fluctuations without lowering the bath temperature.

This work was partially supported by Istituto Nazionale di Fisica Nucleare. S. M. would like to thank F. Marchesoni for suggestions. Discussions with C. Caves, C. Rapagnani, S. Schiller and J. Mlynek are also greatly acknowledged.

[1] V. B. Braginsky and F. Ya Khalili, Quantum Measurements, edited by K. S. Thorne (Cambridge University Press, Cambridge, 1992).

[2] C. M. Caves et al., Rev. Mod. Phys. 52, 341, (1980).

[3] V. B. Braginsky and V. P. Mitrofanov, Systems With Small Dissipation, (University of Chicago Press, Chicago, 1985).

[4] Gravitational Wave Experiments, edited by E. Coccia et al., (World Scientific, Singapore, 1995), see for example: F. J. Raab; A. Giazotto et al., ibid. 
[5] see for example: E. Coccia et al. in Ref. [4]; M. Cerdonio et al., ibid.; P. F. Michelson et al., ibid.

[6] W. Unruh, in Quantum Optics, Experimental Gravitation and Measurements Theory, edited by P. Meystre and M. O. Scully (Plenum, New york, 1983).

[7] H. Casimir, Koninkl. Ned. Akad. Wetenschap. Proc. Ser. B, 793 (1948).

[8] C. K. Law, Phys. Rev. A 51, 2537 (1995).

[9] S. Mancini and P. Tombesi, Phys. Rev. A 49, 4055 (1994).

[10] A. F. Pace et al., Phys. Rev. A 47, 3173 (1993).

[11] P. Tombesi and D. Vitali, Appl. Phys. B 60, S69 (1995).

[12] P. Tombesi and D. Vitali, Phys. Rev. A 50, 4253 (1994).

[13] H.M. Wiseman, and G.J. Milburn, Phys. Rev. A 47, 642 (1993).

[14] H. P. Yuen and J. H. Shapiro, IEEE Trans. IT 26, 78 (1980).

[15] J. Mertz et al., Appl. Phys. Lett. 62, 2344 (1993).

[16] H.M. Wiseman and G.J. Milburn, Phys. Rev. Lett. 70, 548 (1993); Phys. Rev. A 49, 1350 (1994); H.M. Wiseman, Phys. Rev. A 49, 2133 (1994).

[17] A. Barchielli, Phys. Rev. A 34, 1642 (1986).

[18] C.W. Gardiner, Quantum Noise (Springer, Berlin, 1991); see for example Sec. 3.

[19] G. Lindblad, Commun. Math. Phys. 48, 119 (1976).

[20] see for example Sec. 4 of Ref. 18.

[21] see for example Sec. 5 of Ref. 18. 


\section{FIGURE CAPTIONS}

Fig. 1 The quantity $S_{g}(\omega) /\left(2 \pi\left\langle X^{2}\right\rangle_{g=0}\right)$ is plotted (in a semilog scale) versus $\omega$ for the following values of $g$ in $\mathrm{s}^{-1}$ : a) 0 ; b) 1 ; c) 10 ; d) $10^{2}$; e) $10^{3}$. We have used the values of other parameters listed in the text. 